\title{
The Performance of Sphere Decoders for Iterative Spatial Multiplexing MIMO Receiver
}

\author{
Ming Zhao*†, Zhenning Shi ${ }^{\dagger}$, and Mark C. Reed ${ }^{\dagger}$ \\ ${ }^{*}$ Research School of Information Science and Engineering \\ The Australian National University, Canberra, ACT, Australia 0200 \\ ${ }^{\dagger}$ National ICT Australia Ltd, Canberra Research Laboratory \\ Tower A, 7 London Circuit, Canberra, ACT, Australia 2601 \\ Email: \{Ming.Zhao, Zhenning.Shi, Mark.Reed\}@nicta.com.au
}

\begin{abstract}
Pohst and Schnorr-Euchner (SE) are two popular sphere decoders (SD) to perform near maximum likelihood (ML) detection in Spatial Multiplexing multiple-input-multipleoutput (MIMO) system. Recent work [1] showed Pohst and SE are two variants of the well known stack sequential decoding and proposed modification to each strategy to shown significant complexity reduction but with near ML performance. This paper proposed modified Pohst and SE algorithms by taking into account the a priori information to estimate the maximum a posteriori (MAP) probability of the received symbol sequence. Simulation results show that significant performance gain can be achieved from iterative MAP approach and complexity reduction can be obtained for SE enumeration strategy with minor performance loss compared to Phost enumeration algorithm.
\end{abstract}

\section{INTRODUCTION}

Sphere Decoding (SD) [2] has attracted much research attention in performing near maximum likelihood (ML) detection for Multiple-input-multiple-output (MIMO) spatial multiplexing system. The ML detection is optimal compared to the conventional zero forcing $(\mathrm{ZF})$ detector, decision feedback equalizer (DFE), and minimum mean square error (MMSE) detector. However, the complexity of ML detection grows exponentially in terms of the number of antennas. Sphere decoding provides the approximate ML estimate of the transmitted signal sequence by restricting the search range to a limited enumeration set rather than on the completed set of constellation points. It is generally agreed that sphere decoding technique still has polynomial [3] computational complexity far below that of the ML detector.

Pohst enumeration [4] is a well known sphere decoding algorithm to evaluate all the lattice points within a sphere with certain radius. It consists of spanning the tree search by defining a admissible interval at each level. All symbol hypotheses are enumerated at each level between the lower and upper bounds determined by the interval. Nevertheless, Pohst algorithm has a prohibitive complexity which is exponential in the worst case with the dimension of the tree search.

Schnorr-Euchner (SE) enumeration [5] is a variation of the Pohst algorithm. Instead of enumerating all the lattice points within the interval, SE algorithm performs the tree node search in a zig-zag order, starting from the zero forcing solution. Numerical results in [6] showed that SE enumeration is more efficient than the Pohst implementation.
The author in [1] looked at the sphere decoding from the viewpoint of stack sequential decoding algorithm [7] and developed two new sphere decoding algorithms. Algorithm I [1]is based on Phost enumeration by updating the the upper bound at each level to avoid enumerating previously examined partial paths. Algorithm II [1] is to perform SE enumeration with preprocessing and ordering. Numerical results shows significant reductions in the computational complexity with near ML detection performance compared to previously proposed sphere decoding algorithms.

The output of the Phost and SE algorithms in [1] is the lattice point of minimum Euclidean distance to the received signal. This paper proposes modified Pohst and SE algorithms with accumulated a priori information metric to estimate the maximum a posteriori (MAP) probability of the received symbol sequence. We extend the Algorithms I and II in [1] to iterative reception by including accumulated a priori information metric. For Algorithm II, we propose an improved tree search to adjust the starting point in a zig-zag fashion to avoid performing preprocessing and ordering.

This paper is organized as follows. In Section II, the MIMO spatial multiplexing system is described and the iterative detection and decoding are introduced. In Section III, the modified Pohst and SE algorithms employing accumulated a priori information metric are proposed. Simulation results are shown in Section IV. Section V concludes the paper. The notations used in this paper are as follows. Matrices and vectors are denoted by symbols in bold face and $(\cdot)^{*}$, $(\cdot)^{T}$ and $(\cdot)^{H}$ represent complex conjugate, transpose and Hermitian transpose. $E\{\cdot\}$ denotes the statistical expectation. $[\mathbf{X}]_{i, j}$ indicates the $(i, j)$ th elements of a matrix $\mathbf{X}$, and similarly, $\left[\mathbf{x}_{i}\right]$ indicates the element $i$ in a vector $\mathbf{x}$. Finally, $\{x\}$ represents the sequence $x$.

\section{SySTEM MODEL}

\section{A. MIMO Spatial Multiplexing System}

The MIMO spatial multiplexing system considered in this paper is shown in Fig. 1. There are $N_{T}$ transmitting antennas and $N_{R}$ receiving antennas. The information bits $\{\mathbf{b}\}$ are first encoded into coded bits sequences $\{\mathbf{d}\}$. These coded bits are interleaved into a new sequence $\{\mathbf{c}\}$, mapped into a sequence 


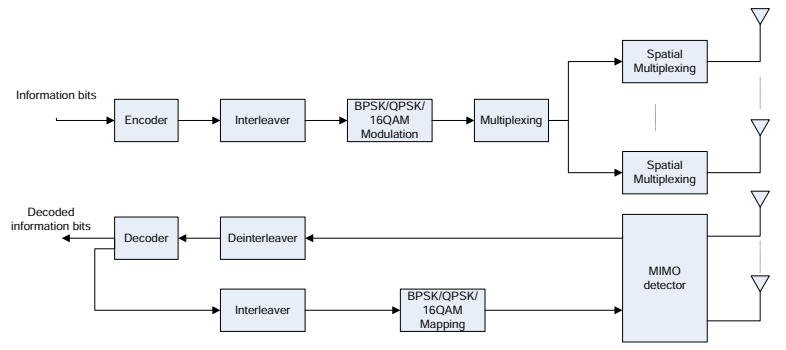

Fig. 1. MIMO spatial multiplexing transmitter with iterative receiver

of $M$-ary complex symbols $\{\mathbf{x}\}$ represented by a vector $\mathbf{x}=$ $\left[x_{0}, x_{1}, \cdots, x_{N_{T}-1}\right]^{T}$.

Assuming that each transmitting and receiving antenna link undergoes independent flat fading, the system model can be expressed as:

$$
\mathbf{y}=\mathbf{h x}+\mathbf{n},
$$

where $\mathrm{y}$ is the received signal arranged as $N_{R} \times 1$ vector $\mathbf{y}=\left[y_{0}, y_{1}, \cdots, y_{N_{R}-1}\right]^{T} . \mathbf{h}$ is $N_{R} \times N_{T}$ channel matrix. $\mathbf{n}$ is $N_{R} \times 1$ additive white Gaussian noise (AWGN) vector with covariance $\sigma^{2} \mathbf{I}_{N_{R}}$. It is convenient to reformulate the system model from a complex value domain to a real value domain by defining the $2 N_{R} \times 1$ vector $\mathbf{Y}, 2 N_{T} \times 1$ vector $\mathbf{X}, 2 N_{R} \times 1$ vector $\mathbf{N}$ as:

$$
\begin{aligned}
& \mathbf{Y}=\left[\begin{array}{ll}
\Re(\mathbf{y})^{T} & \Im(\mathbf{y})^{T}
\end{array}\right]^{T} \\
& \mathbf{X}=\left[\begin{array}{ll}
\Re(\mathbf{x})^{T} & \Im(\mathbf{x})^{T}
\end{array}\right]^{T} \\
& \mathbf{N}=\left[\begin{array}{ll}
\Re(\mathbf{n})^{T} & \Im(\mathbf{n})^{T}
\end{array}\right]^{T}
\end{aligned}
$$

and $2 N_{R} \times 2 N_{T}$ matrix $\mathbf{H}$ as:

$$
\mathbf{H}=\left[\begin{array}{ll}
\Re(\mathbf{h}) & -\Im(\mathbf{h}) \\
\Im(\mathbf{h}) & \Re(\mathbf{h})
\end{array}\right],
$$

where $\Re(\cdot)$ and $\Im(\cdot)$ denote the real and imaginary parts of the argument. Then the system can be expressed in as:

$$
\mathbf{Y}=\mathbf{H X}+\mathbf{N}
$$

\section{B. Iterative Detection and Decoding with Sphere Decoding}

The MIMO detector based on the original Pohst and SE algorithms try to maximize the likelihood of the transmitted signal being detected, that is, to minimize the Euclidian Distance to the received signal given that the channel is known, which is given as:

$$
\hat{\mathbf{X}}=\arg \max _{\mathbf{X} \in \mathcal{A}} p(\mathbf{Y} \mid \mathbf{X})=\arg \min _{\mathbf{X} \in \mathcal{A}}\|\mathbf{Y}-\mathbf{H X}\|^{2},
$$

where $\mathcal{A}$ is the signal set of dimension $2 N_{T}$. In this paper, the modified Phost and SE algorithms aim to estimate the MAP probability of transmitted signal sequence given by:

$$
\hat{\mathbf{X}}=\arg \max _{\mathbf{X} \in \mathcal{A}} p(\mathbf{X} \mid \mathbf{Y})=\arg \max _{\mathbf{X} \in \mathcal{A}} \frac{p(\mathbf{Y} \mid \mathbf{X}) p(\mathbf{X})}{p(\mathbf{Y})} .
$$

In the iterative detection and decoding, $p(\mathbf{X})$ is considered as the a priori information, which is fed back from channel decoder. Because the a priori is not available in the first iteration, sphere decoding based on the ML metric (3) is employed in the MIMO detector in Fig. 1. From the second iteration onwards, MAP detection metric (4) is employed. In each iteration, the MIMO detector selects most reliable points $\{\hat{\mathbf{X}}\}$ from the completed signal constellation set to form a list $\mathcal{U}$ to compute the extrinsic log likelihood ratio (LLR) of the coded bit as:

$$
\begin{aligned}
\lambda_{1}^{e}\left(d_{k}(\hat{\mathbf{X}})\right) & =\frac{1}{2} \sum_{\hat{\mathbf{X}} \in \mathcal{U}_{k}^{+}}\left(-\frac{1}{\sigma^{2}}\|\mathbf{Y}-\mathbf{H} \hat{\mathbf{X}}\|^{2}+\mathbf{d}_{-k}^{T} \lambda_{\mathbf{2},-\mathbf{k}}^{\mathbf{e}}\right) \\
& -\frac{1}{2} \sum_{\hat{\mathbf{X}} \in \mathcal{U}_{k}^{-}}\left(-\frac{1}{\sigma^{2}}\|\mathbf{Y}-\mathbf{H} \hat{\mathbf{X}}\|^{2}+\mathbf{d}_{-k}^{T} \lambda_{\mathbf{2},-\mathbf{k}}^{\mathbf{e}}\right),
\end{aligned}
$$

where $d_{k}(\hat{\mathbf{X}})$ is the $k^{t h}$ coded bit in sequence $\mathbf{d}$ representing the sphere decoder enumerated transmitted symbol vector $\hat{\mathbf{X}}$. $\mathcal{U}_{k}^{+}$and $\mathcal{U}_{k}^{-}$denote the subset of $\mathcal{U}$ for which $d_{k}(\hat{\mathbf{X}})$ is +1 and -1 respectively. $\mathbf{d}_{-k}$ is obtained from sequence $\mathbf{d}$ by removing the $k^{t h}$ coded bit. $\lambda_{2,-k}^{e}$ is the extrinsic LLR of sequence $\mathbf{d}_{-k}$ from the channel decoder. After MIMO detection, the sequence of extrinsic LLR $\left\{\lambda_{1}^{e}\right\}$ of coded bits is deinterleaved and passed through the channel decoder to complete one iteration.

\section{Iterative Pohst ANd SE Sphere Decoder}

Assuming that the transmitted symbols $X_{0}, X_{1} \ldots, X_{2 N_{T}-1}$ are independent, $p(\mathbf{X})$ can be expressed as:

$$
p(\mathbf{X})=\prod_{k=0}^{2 N_{T}-1} p\left(X_{k}\right)=\exp \left(\sum_{k=0}^{2 N_{T}-1} \ln p\left(X_{k}\right)\right) .
$$

Hence, (4) can be reformulated as:

$$
\begin{aligned}
\hat{\mathbf{X}} & \approx \arg \max _{\mathbf{X} \in \mathcal{A}} p(\mathbf{Y} \mid \mathbf{X}) p(\mathbf{X}) \\
& \approx \arg \max _{\mathbf{X} \in \mathcal{A}} \exp \left(-\frac{\|\mathbf{Y}-\mathbf{H X}\|^{2}}{2 \sigma^{2}}+\sum_{k=0}^{2 N_{T}-1} \ln p\left(X_{k}\right)\right) \\
& \approx \arg \min _{\mathbf{X} \in \mathcal{A}}\left(\|\mathbf{Y}-\mathbf{H X}\|^{2}-2 \sigma^{2} \sum_{k=0}^{2 N_{T}-1} \ln p\left(X_{k}\right)\right) .
\end{aligned}
$$

The modified Pohst and SE algorithms narrow down the search region as:

$$
\|\mathbf{Y}-\mathbf{H X}\|^{2}-2 \sigma^{2} \sum_{k=0}^{2 N_{T}-1} \ln p\left(X_{k}\right) \leq C .
$$

By performing QR decomposition, the channel matrix $\mathbf{H}$ can be expressed as:

$$
\mathbf{H}=\left[\begin{array}{ll}
\mathbf{Q} & \mathbf{Q}^{\prime}
\end{array}\right]\left[\begin{array}{c}
\mathbf{R} \\
\mathbf{0}
\end{array}\right] .
$$

where $\mathbf{Q}$ is a $2 N_{R} \times 2 N_{T}$ unitary matrix, $\mathbf{Q}^{\prime}$ is $2 N_{R} \times\left(2 N_{R}-\right.$ $\left.2 N_{T}\right)$ unitary matrix, $\mathbf{R}$ is a $2 N_{T} \times 2 N_{T}$ upper triangular matrix, and $\mathbf{0}$ is $\left(2 N_{R}-2 N_{T}\right) \times 2 N_{T}$ zero matrix. Known that:

$$
\left[\begin{array}{ll}
\mathbf{Q} & \mathbf{Q}^{\prime}
\end{array}\right]^{H}\left[\begin{array}{ll}
\mathbf{Q} & \mathbf{Q}^{\prime}
\end{array}\right]=\mathbf{I}_{2 N_{R}}
$$


Take (9) into (8), we have:

$$
\begin{array}{r}
\left\|\left[\begin{array}{ll}
\mathbf{Q} & \mathbf{Q}^{\prime}
\end{array}\right](\mathbf{Y}-\mathbf{H X})\right\|^{2}-2 \sigma^{2} \sum_{k=0}^{2 N_{T}-1} \ln p\left(X_{k}\right) \leq C \\
\left\|\mathbf{Q}^{H} \mathbf{Y}-\mathbf{R X}\right\|^{2}-2 \sigma^{2} \sum_{k=0}^{2 N_{T}^{-1}} \ln p\left(X_{k}\right) \leq C \\
\left\|\mathbf{Y}^{\prime}-\mathbf{R X}\right\|^{2}-2 \sigma^{2} \sum_{k=0}^{2 N_{T}-1} \ln p\left(X_{k}\right) \leq C-\left\|\mathbf{Q}^{\prime H} \mathbf{Y}\right\|^{2}
\end{array}
$$

where $\mathbf{Y}^{\prime}=\mathbf{Q}^{H} \mathbf{Y}$.

By expanding the term $\left\|\mathbf{Y}^{\prime}-\mathbf{R X}\right\|^{2}$, we can further simplify (11) as follows:

$$
\sum_{k=i}^{2 N_{T}-1}\left|Y_{k}^{\prime}-\sum_{j=k}^{2 N_{T}-1} R_{k, j} X_{j}\right|^{2}-2 \sigma^{2} \sum_{k=0}^{2 N_{T}-1} \ln p\left(X_{k}\right) \leq C^{\prime}
$$

where $C^{\prime}$ is the newly defined sphere radius.

\section{A. MIMO detector with modified Pohst algorithm}

Given $\mathbf{Y}^{\prime}, \mathbf{R}$, the a priori information $p(\hat{\mathbf{X}})$, and the radius $C^{\prime}$, the modified Pohst algorithm can be outlined as follows:

1) Set tree search level index $i:=2 N_{T}-1$, path metric $\vartheta_{i}:=0$, branch metric $\zeta_{i}:=0$, accumulated a priori information metric $\delta_{i}:=0$, constellation index $\lambda_{j}:=$ $0, j \in\left[0,2 N_{T}-1\right]$, constellation set $\Phi$, and current radius $d:=C^{\prime}$.

2) If $\left(d<\vartheta_{i}\right)$

go to step 4 .

Else \{

compute the lower bound $\mathcal{L}_{i}:=\left\langle\frac{Y_{i}^{\prime}-\zeta_{i}-\sqrt{d-\vartheta_{i}}}{R_{i, i}}\right\rangle$, compute the upper bound $\mathcal{V}_{i}:=\left\langle\frac{Y_{i}^{\prime}-\zeta_{i}+\sqrt{d-\vartheta_{i}}}{R_{i, i}}\right\rangle$, $\lambda_{i}:=i d x\left(\mathcal{L}_{i}, \Phi\right)-1, i d x(\cdot)$ is the operator that finds the index in constellation set $\Phi$, and $\langle\cdot\rangle$ is operator that finds the nearest constellation point. $\}$

3) $\lambda_{i}:=\lambda_{i}+1, \hat{X}_{i}:=\Phi\left(\lambda_{i}\right)$

If $\left(\hat{X}_{i} \leq \mathcal{V}_{i}\right)$

$X_{i}$ within the interval, go to step 5 .

Else

$\hat{X}_{i}$ outside the interval, go to step 4 .

4) If ( $\left.i==2 N_{T}-1\right)$

go to step 7.

Else

$i:=i+1$, go to step 3 .

5) If $(i>0)\{$

update branch metric $\zeta_{i-1}:=\sum_{k=i}^{2 N_{T}-1} R_{i-1, k} \hat{X}_{k}$,

update accumulate a priori information metric:

$$
\delta_{i}:=\delta_{i-1}+2 \sigma^{2} \ln p\left(\hat{X}_{i}\right)
$$

update path metric:

$$
\vartheta_{i-1}:=\vartheta_{i}+\left\|Y_{i}^{\prime}-\zeta_{i}-R_{i, i} \hat{X}_{i}\right\|^{2}-\delta_{i},
$$

$i:=i-1$, go to step 2 . $\}$

Else if $(i==0)$

go to step 6.
6) If $\left(d>\vartheta_{i}+\left\|Y_{i}^{\prime}-\zeta_{i}-R_{i, i} \hat{X}_{i}\right\|^{2}-\delta_{i}\right)\{$

updating the radius $d:=\vartheta_{i}+\left\|Y_{i}^{\prime}-\zeta_{i}-R_{i, i} \hat{X}_{i}\right\|^{2}-\delta_{i}$, save $\hat{\mathbf{X}}$ in candidate list $\mathcal{U}$,

go one level up $i:=i+1$, go to step 7.\}

7) If ( $C^{\prime}$ is within Upper bound)

If(List has less points as required) Else

Increase the radius $C^{\prime}$, go to step 1 .

Terminate.

The proposed algorithm differs from the original Pohst algorithm in that it includes a priori information metric $\delta_{i}$ accumulated along the way of tree search. If the visited nodes diverge from the transmitted signal, $\delta_{i}$ is likely to tend large, which in turn leads to an increasing path metric $\vartheta_{i}$ and a dramatically reduced search radius in $(i+1)^{t h}$ level. This is because the lower bound $\mathcal{L}_{i}$ and upper bound $\mathcal{V}_{i}$ in step 2 are adjusted to a smaller admissible interval such that further search will be limited within a much smaller interval. Therefore, the resultant search path from $i^{\text {th }}$ level will be identified and pruned at an early stage, and the number of tree nodes visited will be reduced.

\section{B. MIMO detector with modified SE algorithm}

Given $\mathbf{Y}^{\prime}, \mathbf{R}$, the a priori information $p(\hat{\mathbf{X}})$, and the radius $C^{\prime}$, the modified SE algorithm can be outlined as follows:

1) Set tree search level index $i:=2 N_{T}-1$, path metric $\vartheta_{i}:=0$, branch metric $\zeta_{i}:=0$, accumulated a priori information metric $\delta_{i}:=0$, zig-zag search index $\lambda_{j}:=$ $0, j \in\left[0,2 N_{T}-1\right]$, radius increasing index $\Delta:=0$, and current radius $d:=C^{\prime}$.

2) If (the node is first time visited) \{

Compute the ZF-DFE estimate of transmitted symbol at level $i$ as $\hat{X}_{i}=\left\langle\left(Y_{i}^{\prime}-\zeta_{i}\right) / R_{i, i}\right\rangle$, where $\langle\cdot\rangle$ is operator that finds the nearest constellation point. Create zig-zag lookup table $\Phi_{i}$ for constellation point $\hat{X}_{i}$. Define $|\Phi|$ as the number of possible zig-zag points.

$$
\begin{aligned}
& \text { If }\left(i==2 N_{T}-1\right) \\
& \quad \lambda_{i}:=\lambda_{i}+\Delta+1, X_{i}=\Phi_{i}\left(\lambda_{i}\right) .
\end{aligned}
$$

Compute the accumulated a priori information metric $\left.\delta_{i}:=\delta_{i-1}+2 \sigma^{2} \ln p\left(\hat{X}_{i}\right).\right\}$

Else If $\left(\lambda_{i}==\left|\Phi_{i}\right|-1\right)$

$$
\text { If }\left(i==2 N_{T}-1\right)
$$$$
\text { go to step } 7 .
$$

$$
\text { Else }
$$$$
\lambda_{i}:=0, \hat{X}_{i}=\Phi_{i}\left(\lambda_{i}\right), i:=i+1, \text { go to step } 6 .
$$

Else

go to step 6.

3) If (the current radius is less than the path metric at level $i$, i.e. $\left.d<\vartheta_{i}+\left\|Y_{i}^{\prime}-\zeta_{i}-R_{i, i} \hat{X}_{i}\right\|^{2}-\delta_{i}\right)$

Out of sphere, go to step 4.

Else If $(i>0)\{$

update branch metric:

$$
\zeta_{i-1}:=\sum_{k=i}^{2 N_{T}-1} R_{i-1, k} \hat{X}_{k}
$$

update path metric: 


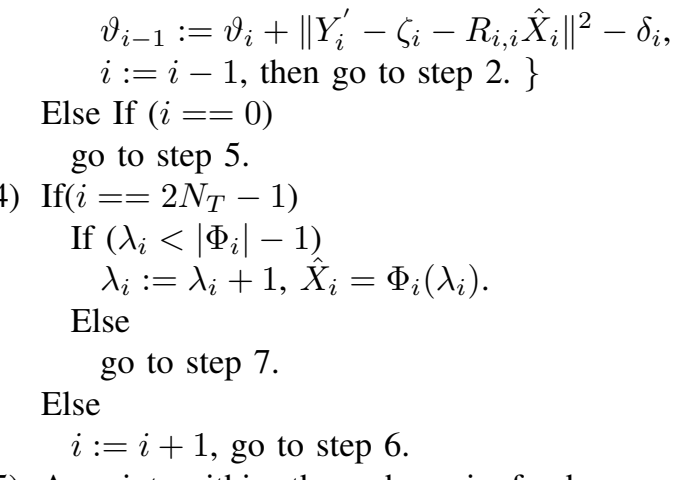

5) A point within the sphere is fund, save $\hat{\mathbf{X}}$ in the candidate list $\mathcal{U}$. Updating the radius $d:=\vartheta_{i}+\| Y_{i}^{\prime}-$ $\zeta_{i}-R_{i, i} \hat{X}_{i} \|^{2}-\delta_{i}$, then go one level up $i:=i+1$, go to step 6 .

6) $\lambda_{i}:=\lambda_{i}+1$, while $\left(\lambda_{i}>\left|\Phi_{i}\right|-1\right)\{$
$\lambda_{i}:=0, i:=i+1$.
If $\left(i>2 N_{T}-1\right)$
$\quad$ go to step 7.
$\left.\quad \lambda_{i}:=\lambda_{i}+1.\right\}$
$\hat{X}_{i}=\Phi_{i}\left(\lambda_{i}\right), \delta_{i}:=\delta_{i-1}+2 \sigma^{2} \ln p\left(\hat{X}_{i}\right)$, go to step 3.

7) If ( $C^{\prime}$ is within Upper bound)

If(List has less points as required) Else

Increase the radius $C^{\prime}, \Delta:=\Delta+1$, go to step 1 .

Terminate.

Unlike the modified Pohst algorithm using the lower and upper boundary adaption to prune the less likely path, the modified SE algorithm eliminates the path by making the comparison between the current available sphere radius and the path metric. A large path metric $\vartheta_{i}$ is more likely to be outside the available sphere radius. Hence, further search starting from such less reliable enumerated node to its children nodes will not be probable.

Another salient feature of the modified SE algorithm is the ZF-DFE in step 2. As discussed in [1], in the original SE algorithm, the ZF-DFE estimate of transmitted signal at $i^{t h}$ level depends on the branch metric only. Such approach may end up with less reliable estimation, which will affect the convergence of the tree search and introduce performance loss. [1] proposed preprocessing and ordering to improve ZF-DFE estimate, which is more complex. Our approach is to make a trial on another constellation point rather than the ZF-DFE estimate. We propose to do this on a zig-zag basis to visit the neighboring constellation point of ZF-DFE estimate.

\section{Simulation Results}

\section{A. Simulation Setup}

We consider a $4 \times 4$ MIMO spatial multiplexing system. The channel model for each transmit and receive antenna are independent flat Rayleigh fading channel. A rate- $1 / 2(171,133)_{8}$ convolutional code is used for channel coding. Each block of information bits has the length of 9216. The modulation includes QPSK and 16QAM. We compared the Bit Error Rate
(BER) and complexity for the non-iterative receivers with original algorithms and iterative receivers with modified sphere decoders. We refer to the original Pohst and SE algorithm in [1] as "Pohst ML" and "SE ML" respectively, the modified Pohst algorithm in III-A as "Pohst MAP", and the modified SE algorithm in III-B as "SE MAP". The computation load is measured by the number of visiting nodes in the tree search, which dominates the system complexity [3] and indicates the convergence speed of algorithms.

\section{B. Numerical Results}

Fig. 2 shows the BER performance for original Pohst and SE algorithms, and the modified Pohst and SE algorithms after 4 iterations. It can be observed that the proposed sphere decoding algorithms have a $2 \mathrm{~dB}$ gain for QPSK modulation, and $3 \mathrm{~dB}$ gain for 16QAM modulation over the original sphere coding algorithms at BER level of $10^{-4}$. This observation suggests that the iterative sphere decoding can achieve significant performance achievement over the generic sphere decoders for the non-iterative receivers. Regarding the performance of different sphere decoding algorithms, it can be seen that the original Pohst algorithm outperforms the original SE algorithm by $1 \mathrm{~dB}$. This difference has been reduced to $0.5 \mathrm{~dB}$ between the two modified algorithms in the iterative reception.

Fig. 3 shows computation complexity for the modified Pohst and SE algorithms over SNRs with 16QAM modulation. It can be shown that Pohst algorithm generally has higher complexity than the SE algorithm. This is because the Pohst algorithm enumerates all the points within the admissible interval, while SE algorithm only searchs the points around the ZF-DFE estimate. Therefore, a favorable complexity reduction can be obtained by the modified SE algorithm with minor performance loss compared to modified Phost algorithm shown in Fig. 2. As expected, by employing the a priori information, the complexity of modified algorithms can be further reduced over iterations because the additional priori information metric

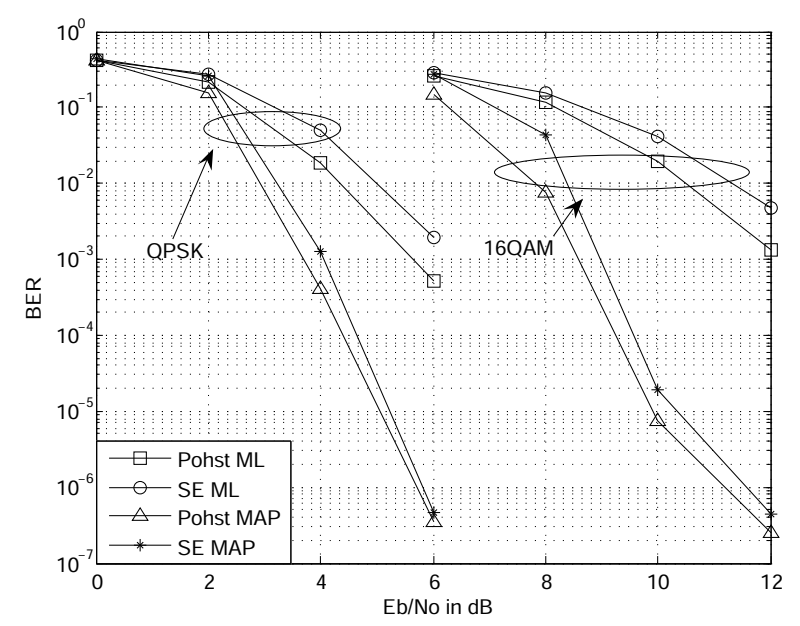

Fig. 2. BER performance for original Pohst and SE algorithms, modified Pohst algorithm, and modified SE algorithms in a $4 \times 4$ MIMO spatial multiplexing system with QPSK and 16QAM modulation 


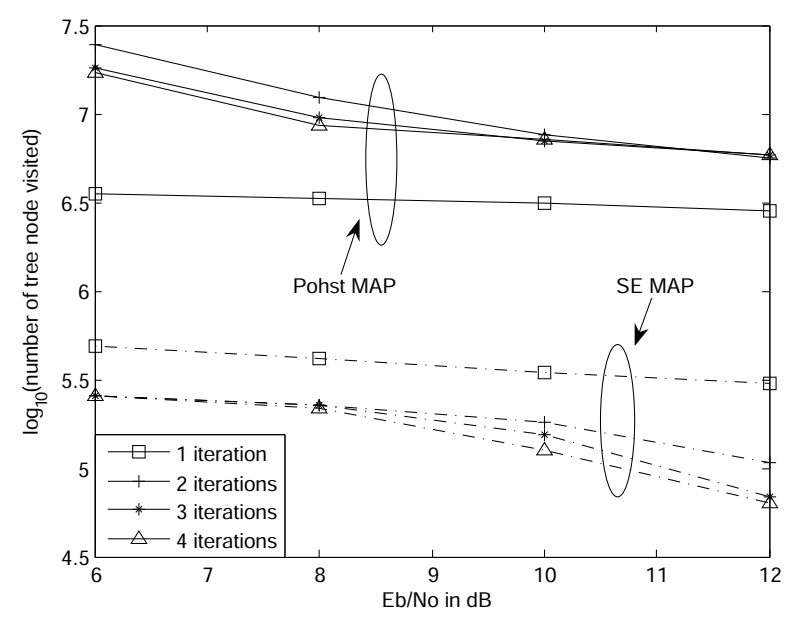

Fig. 3. Computation complexity for modified Pohst algorithm and modified SE algorithms over SNRs in a $4 \times 4$ MIMO spatial multiplexing system with 16QAM modulation

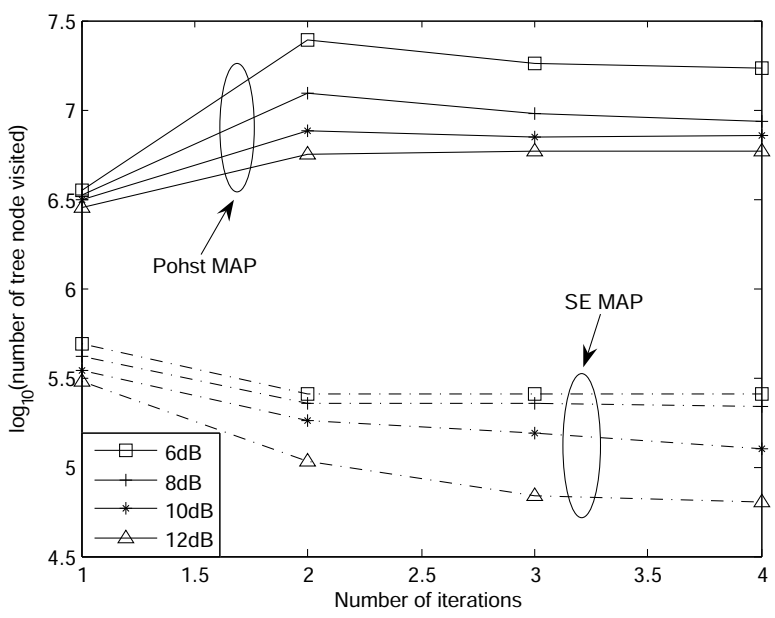

Fig. 4. Computation complexity for modified Pohst algorithm and modified $\mathrm{SE}$ algorithms over iterations in a $4 \times 4$ MIMO spatial multiplexing system with 16QAM modulation

improves the search by adjusting the overall path metric according to the reliability of the enumerated points in addition to the Euclidian Distance. It helps identify and discard the inaccurate search path so that the unnecessary search efforts can be saved.

Fig. 4 shows the computation complexity for the modified Pohst and SE algorithms over iterations with 16QAM modulation. It is interesting to notice that for the modified Pohst algorithm, the complexity of the second iteration is higher than that for the first iteration in the low SNR region, and this difference is getting smaller as SNR goes higher. However, this is not the case for the modified SE algorithm. This is because in the modified Pohst algorithm, the enumeration always starts from the lower bound and ends at higher bound. At low SNR, after the first iteration, the a priori information is not reliable, which leads to a loose lower and upper bounds and slows down the tree search. Such effect becomes marginal with more stable priori information at high SNRs as show in Fig. 4. On the other hand, for the modified SE algorithm, the zig-zag tree search is started from point around the ZF-DFE point, which is independent to the unreliable a priori information after the first iteration.

\section{CONClusion}

In this paper, we proposed modified Pohst and SE algorithm with accumulated a priori information metric for iterative spatial multiplexing MIMO receiver. The original Pohst and SE algorithms in [1] is modified to estimate the MAP probability of transmitted signal sequence. Performance comparison shows significant performance gain over non-iterative receiver with ML metric can be obtained, and complexity reduction can be obtained from SE enumeration strategy with minor performance loss compared to Phost enumeration algorithm.

\section{ACKNOWLEDGMENT}

M. Zhao, Z. Shi and M. C. Reed are with National ICT Australia and affiliated with the Australian National University. National ICT Australia is funded through the Australian Governments backing Australia's Ability initiative and in part through the Australia Research Council.

\section{REFERENCES}

[1] M. Damen, H. Gamal, and G. Caire, "On maximum-likelihood detection and the search for the closest lattice point," IEEE Trans. Inform. Theory, vol. 49, no. 10, pp. 2389-2402, Oct 2003.

[2] E. Viterbo and J. Boutros, "A universal lattice code decoder for fading channels," IEEE Trans. Inform. Theory, vol. 45, pp. 1639-1642, July 1997.

[3] B. Hassibi and H. Vikalo, "On the sphere-decoding algorithm I. expected complexity," IEEE Trans. Signal Processing, vol. 53, no. 8, pp. $2806-$ 2818, Aug 2005.

[4] U. Fincke and M. Pohst, "Improved methods for calculating vectors of short length in a lattice, including a complexity analysis," Math.Comput., vol. 44, no. 4, pp. 463-471, Apr 1985.

[5] C. P. Schnorr and M. Euchner, "Lattice basis reduction: Improved pratical algorithms and solving subset sum problems," Math.Programming., vol. 66, pp. 181-191, 1994.

[6] E. Agrell, T. Eriksson, A. Vardy, and K. Zeger, "Cloest point search in lattices," IEEE Trans. Inform. Theory, vol. 45, pp. 2201-2214, Aug 2002.

[7] A. Viterbi and J. Omura, Principles of Digital Communications and Coding. McGraw-Hill, 1979. 